FE DE ERRORES

\title{
Cuidado de la salud oral en la primera infancia: La perspectiva de sus madres-un estudio cualitativo.
}

\section{Oral health care in early childhood: mother's perspective-a qualitative research.}

\author{
Paulina Humeres-Flores ${ }^{1 *}$, Daniela Guzmán-Orellana ${ }^{1}$, \\ Carla Madrid-Canales ${ }^{1}$, Amanda Fredes-Ziliani ${ }^{2}$, Alexandra Mustakis-Truffello ${ }^{1}$
}

1. Pontificia Universidad Católica de Chile Facultad de Medicina - Escuela de Odontología. Santiago, Chile.

2. Servicio de Salud Metropolitano Sur Oriente: Centro de Salud Familiar Malaquías Concha - La Granja. Santiago, Chile.

* Correspondencia Autor: Dra. Paulina Humeres | Dirección: Av. Vicuña Mackenna 4860, Macul campus San Joaquín - Escuela de Odontologia. Santiago, Chile.|E-mail: phumeres@uc.cl | Teléfono: +56998259323 - +562 23548400 Trabajo recibido el 05/02/2020

Aprobado para su publicación el 28/06/2020
Se hace constar que el manuscrito es fruto de una investigación realizada por las autoras señaladas en el documento, como consecuencia del concurso interno realizado por la Escuela de Odontologì UC el 2016 y cuenta con valiosos aportes de las personas que agradecemos en el apartado correspondiente, sin relacionarse en absoluto con mi formación doctoral, ni con mi tesis de investigación.

Sin perjuicio de ello, el director me instó a poner dentro de mi identificación mi calidad de candidata a doctora a comunicaciones por la Facultad de Comunicaciones UC, porque es de hecho mi condición desde abril del 2019. En consecuencia, agradecería que puedan agregar este aspecto en mi identificación como autora.

Además, el equipo de investigadoras aceptó agregar a los agradecimientos a la profesora Rayén Condeza, el texto que ella pide. Es así, que, a continuación de su nombre, debe ir, "PhD. Profesora Asociada Facultad de Comunicaciones UC (Chile). 